\title{
Synthesis and Properties of Cross-linkable Waterborne
}

\section{Polyurethane/HMMM-CNT Nanocomposite}

\author{
Mohammad Mizanur Rahman ${ }^{1^{*}}$, M. Nasiruzzaman Shaikh ${ }^{2}$ \\ ${ }^{1}$ Center of Research Excellence in Corrosion Science, ${ }^{2}$ Center of Research Excellence in Nano \\ Technology, King Fahd University of Petroleum and Minerals, Dhahran-31261, Saudi Arabia. \\ Email: mrahman@kfupm.edu.sa ("correspondence author), Tel: + 966-13-860-7210.
}

Keywords: Polyurethane, Crosslinking, CNT, Melamine.

\begin{abstract}
A series of cross-linked waterborne polyurethane/hexamethoxymethylmel-amine-carbon nanotube nanocomposites (WBPU/HMMM-CNT) were synthesized using carboxylic group functionalized CNT. The carboxylic groups on CNT were reacted with the methoxy groups of HMMM to get bonded HMMM-CNT. Unreacted methoxy group of HMMM-CNT was crosslinked with the carboxylic acid salt groups of WBPU and made crosslinked WBPU/HMMM-CNT nanocomposite. The mechanical properties (tensile strength and Young's modulus) of conventional WBPU, crosslinked WBPU/HMMM, WBPU/CNT and WBPU/HMMM-CNT nanocomposites were compared under three conditions: untreated, wet and dried. It was observed that the mechanical properties of the crosslinked WBPU/HMMM-CNT nanocomposites were the least affected by water compared to conventional WBPU, crosslinked WBPU/HMMM, and WBPU/CNT nanocomposites. Differential scanning calorimetry (DSC) analysis also confirmed that the WBPU/HMMM-CNT nanocomposite can absorbed least water which can be easily removed by heating without destroying their crystalline structure. Crosslinked WBPU/HMMM-CNT nanocomposite recovered most of its mechanical properties of (with optimum HMMM-CNT content) after drying.
\end{abstract}




\section{Introduction}

Waterborne polyurethane (WBPU) is an important environmentally friendly polymer since only water is evolved during the drying stage. This material has a wide range of applications in construction, automotive, packaging, transportation, electronics, textiles, tape, paper, and footwear industries [1-5]. Unfortunately WBPU is inferior with respect to chemical resistance, thermal resistance, and mechanical properties (tensile strength and initial modulus) as compared with solvent-based polyurethanes [2-4]. There have been many studies that address some of these disadvantages. To minimize the above disadvantages significant efforts were made during the last decade using various approaches. Kwak et al. [6] studied the effect of dimethylol propionic acid (DMPA) content on the tensile strength and adhesive strength of WBPU. They showed that tensile strength and adhesive strength increases with increasing DMPA content. Yang et al. [7] studied the effects of different types of neutralizing agents on the tensile strength and adhesive strength of polycaprolactone based WBPU. The tensile strength and adhesive strength was maximized when the DMPA was fully neutralized during the preparation of WBPU material. Kim and Kim [8] studied the tensile strength, water resistance and adhesive strength of various polyester polyol based WBPU. In our previous work [1], we were able to improve the tensile strength, water resistance and adhesive strength of WBPU in several ways, such as increasing DMPA content, using polytetramethylene adipate glycol [1, 2], incorporating an aliphatic hardener [9] and making nanocomposites [10-12].

Recently, WBPU/CNT nanocomposites have attracted much attention owing to their industrial importance. They have shown a dramatic improvement in mechanical (tensile strength and Young's modulus), thermal, and barrier properties with the addition of a small amount of CNT in the composites [11]. However, major challenges remain in WBPU/CNT nanocomposites are related to CNT dispersion via industrially scalable, environmentally friendly methods and understanding the relationship between dispersion and optimal properties. Several strategies have been studied to achieve well-dispersed WBPU/CNT nanocomposites, including surface functionalization and/or sonication pretreatment, higher DMPA content in WBPU, and in situ polymerization [10-12]. 
Crosslinking is another promising technique for increasing the tensile strength, water resistance and adhesive strength of WBPU $[4,5]$. Hexamethoxymethylmelamine (HMMM), in the presence of catalyst, can cross-linked to hydroxyl, carboxyl, and amide groups present in alkyds, polyesters, acrylics, epoxies, and urethanes $[13,15]$. It has many advantages, like, the crosslinking process is economical, fast, solvent free, stable, and widely compatible with other polymers. Additionally, the cross linker is soluble in various resins and the resulting WBPU has excellent hardness and film flexibility [4].

Polyurethanes are being used in marine boats, vessels, ships, and offshore drilling platforms for deck grates as coatings to provide waterproof surfaces. These coatings undergo much wear and tear from being in water and being subjected to various weather conditions. These polyurethane coatings are synthesized using large amount of organic volatile solvents. Nowadays this type of polyurethane is being restricted due to emission of harmful organic moieties. People are searching for an alternative coating material which can fulfill the adequate water resistivity with sufficient mechanical strength and the synthesis scheme follows the environmental regulations (less organic volatile solvent). Thus interests grow on environment friendly WBPU coating for this sector. Unfortunately, there have not been any systematic analyses of these WBPU, cross- linkable WBPU and WBPU nanocomposites under adverse conditions such as after dipped in water or after dried the films. In this report, we synthesized cross-linked WBPU/HMMM-CNT nanocomposite and compared the properties (mechanical properties, glass transition temperature $(T \mathrm{~g})$ and water resistance) with conventional WBPU, cross-linkable WBPU/HMMM and WBPU/CNT nanocomposite. The mechanical properties (tensile strength, and Young's modulus) were also compared under three conditions: untreated, wet and dried. The differential scanning calorimetry (DSC) was also applied to characterize the polymer under these three conditions. 


\section{Experimental}

Materials. Terathane 2000 (Sigma Aldrich) was dried under vacuum at $1-2 \mathrm{mmHg}$ at $90{ }^{\circ} \mathrm{C}$ for $3 \mathrm{~h}$ before use. Triethylamine (TEA; Junsei Chemical, Tokyo, Japan), N-methyl-2-pyrrolidone (NMP; Junsei Chemical), 4,4'-methylenebis(cyclohexylisocyanate) ( $\mathrm{H}_{12} \mathrm{MDI}$; Sigma Aldrich), ethylene diamine (EDA; Junsei Chemical) and methyl ethyl ketone (MEK; Junsei Chemical), were dehydrated with 4- $\AA$ molecular sieves for 1 week before use. 2,2-Bis(hydroxymethyl)propionic acid (DMPA, Sigma Aldrich), dibutyltin dilaurate (Sigma Aldrich), hexamethoxymethylmelamine (HMMM; TCI) and $p$-toluene sulphonic acid (Sigma Aldrich) were used as received. The multiwalled CNT (average diameter $=15 \mathrm{~nm}$, thermal chemical vapor deposited material; Iljin Nanotech Co. Ltd., Korea) was used after functionalization by nitric acid and sulphuric acid treatment [10].

Preparation of the Acid Functionalized CNT. The multi-walled CNTs were functionalized according to our previous reports $[10,12]$. At first the raw CNTs were washed with 20\% hydrofluoric acid $(5 \mathrm{~h})$ and $22 \%$ nitric acid $(10 \mathrm{~h})$ and then subsequently washed with distilled water until the $\mathrm{pH}$ of the CNTs (in water) approached 7. The washed CNTs were treated with boiled nitric acid for a period of $0.5 \mathrm{~h}$ and the acid functionalized CNTs were washed with distilled water and acetone.

Preparation of the HMMM-CNT Dispersion. The HMMM-CNT dispersion was prepared by treating HMMM and functionalized CNT with $p$-toluene sulphonic acid catalyst. HMMM (5 g) was dissolved in water $(20 \mathrm{ml})$ by sonication for 30 minutes. The functionalized CNT (1 g) was separately dispersed in water $(20 \mathrm{ml})$ by sonication for 10 minutes and added to the HMMM solution. The temperature of the mixture was maintained at $50{ }^{\circ} \mathrm{C}$. The catalyst $p$-toluene sulphonic acid (0.01 g, dissolved in MEK $5 \mathrm{~g}$ ) was added to the reaction mixture. The reaction ran for 1 hour and was monitored by FT-IR spectroscopy. 
Preparation of WBPU Dispersion. WBPU dispersion was prepared as described in our previous report [1]. Terathane was degassed under vacuum at $90{ }^{\circ} \mathrm{C}$ for $30 \mathrm{~min}$ in a four-necked separating flask equipped with a thermometer, stir bar, condenser with drying tube, dry nitrogen inlet, and heat jacket. DMPA/NMP $(1 / 1 \mathrm{w} / \mathrm{w})$ was added to the flask, and the mixture was allowed to cool to $45{ }^{\circ} \mathrm{C}$ under moderate stirring (175 to $200 \mathrm{rpm}$ ). $\mathrm{H}_{12} \mathrm{MDI}$ and DBTDL were added to the mixture and heated to $85{ }^{\circ} \mathrm{C}$ under the same stirring rate (175 to $\left.200 \mathrm{rpm}\right)$. The reaction was allowed to continue at these conditions for $3 \mathrm{~h}$. The change in the NCO value during the reaction was determined by the standard dibutylamine back-titration method (ASTM D 1638). Methyl ethyl ketone (MEK, 10 wt\%) was added to the NCO-terminated prepolymer mixture to decrease the viscosity of the reaction mixture. TEA was added to the reaction mixture at $65{ }^{\circ} \mathrm{C}$ to neutralize the carboxyl group of the NCO-terminated prepolymer. After $30 \mathrm{~min}$ of neutralization, distilled water (70 $\mathrm{wt} \%)$ was added to the reaction mixture with vigorous stirring (1300 to $1500 \mathrm{rpm}$ ). The resulting dispersion was chainextended by dropwise addition of EDA (with water) at $40{ }^{\circ} \mathrm{C}$ for $1 \mathrm{~h}$. The reaction was allowed to continue until the NCO peak $\left(2260-2280 \mathrm{~cm}^{-1}\right)$ in the IR spectra had disappeared completely. The WBPU dispersions (30 wt\% solid content) were obtained after evaporation of MEK.

Preparation of the WBPU/CNT Nanocomposite Dispersions. WBPU/CNT nanocomposite dispersions were prepared as described in our previous report [12]. Terathane was degassed under vacuum at $90{ }^{\circ} \mathrm{C}$ for $30 \mathrm{~min}$ in a four-necked separating flask equipped with a thermometer, stir bar, condenser with drying tube, dry nitrogen inlet, and heat jacket. DMPA/NMP $(1 / 1 \mathrm{w} / \mathrm{w})$ was added to the flask, and the mixture was allowed to cool to $45^{\circ} \mathrm{C}$ under moderate stirring $(175-200 \mathrm{rpm})$. The $\mathrm{H}_{12} \mathrm{MDI}$ and DBTDL were added to the mixture at $45^{\circ} \mathrm{C}$ and heated to $85^{\circ} \mathrm{C}$ under similar stirring rate $(175-200 \mathrm{rpm})$. The reaction mixture was allowed to react at $85{ }^{\circ} \mathrm{C}$ for $3 \mathrm{~h}$. The change in the NCO value during the reaction was determined with the standard dibutylamine back-titration method (ASTM D 1638). Then, MEK (10 wt $\%$ ) was added to the NCO-terminated prepolymer mixture to decrease the viscosity of the reaction mixture. TEA was added to the reaction mixture at $65{ }^{\circ} \mathrm{C}$ to neutralize the carboxyl group of the NCO-terminated polyurethane prepolymer. After 30 min of 
neutralization, distilled water $(70 \mathrm{wt} \%)$ was added to the reaction mixture with vigorous stirring (1300-1500 rpm). The CNT-water dispersion was prepared according to our previous reports $[10,12]$ and added in the dispersion step. The WBPU/CNT dispersion was chain-extended by dropping EDA/ $\mathrm{H}_{2} \mathrm{O}$ mixture at $40{ }^{\circ} \mathrm{C}$ for $1 \mathrm{~h}$, and the reaction continued until the NCO peak (2260$2280 \mathrm{~cm}^{-1}$ ) in the IR spectra had disappeared completely. After evaporation of MEK, the WBPU/CNT nanocomposite dispersions were obtained.

Preparation of the Cross-linkable WBPU/HMMM Dispersion. Crosslinked WBPU/HMMM dispersion was prepared as described in our previous report [5]. The neutralized prepolymer was prepared as described in our previous report for conventional WBPU dispersion [5]. Distilled water (70 $\mathrm{wt} \%$ ) was then added to the reaction mixture under vigorous stirring (1300 to $1500 \mathrm{rpm}$ ). The catalyst, $p$-toluene sulfonic acid (dissolved in MEK), and HMMM (dissolved in water) were separately added to the dispersion at $50{ }^{\circ} \mathrm{C}$. EDA (in water) was added dropwise to the dispersion at $40{ }^{\circ} \mathrm{C}$ for $1 \mathrm{~h}$, and the reaction was allowed to continue until the NCO peak $\left(2260-2280 \mathrm{~cm}^{-1}\right)$ in the IR spectra had disappeared completely. The crosslinked WBPU/HMMM dispersions (30 wt $\%$ solid content) were obtained after evaporation of MEK.

Preparation of the Cross-linked WBPU/HMMM-CNT Nanocomposite Dispersions. The crosslinked WBPU/HMMM-CNT nanocomposite dispersions were prepared by a prepolymer process. The neutralized prepolymer was prepared in the same manner as that of WBPU dispersion. The distilled water $(70 \mathrm{wt} \%)$ was added to the reaction mixture with vigorous stirring (1300 to 1500 rpm). The catalyst $p$-toluene sulphonic $(0.01 \mathrm{~g})$ acid (dissolved in MEK $5 \mathrm{~g}$ ) and HMMM-CNT dispersion were added separately at $50{ }^{\circ} \mathrm{C}$ to WBPU dispersion and mixed for 1 hour. The EDA (in water) was added to the dispersion at $40{ }^{\circ} \mathrm{C}$, and the reaction continued until the NCO peak (2260$2280 \mathrm{~cm}^{-1}$ ) in the IR spectra had disappeared completely. The crosslinked WBPU/HMMM-CNT nanocomposite dispersions (30 wt \% solid content) were obtained after evaporation of MEK. 


\section{Preparation of the WBPU, WBPU/CNT, WBPU/HMMM, WBPU/HMMM-CNT}

Nanocomposite Films. The polymer films were prepared by drying the aqueous dispersions (WBPU, WBPU/CNT, WBPU/HMMM, WBPU/HMMM-CNT $10 \mathrm{~g}$ ) on a Teflon disk (diameter 7 $\mathrm{cm}$ ) under ambient conditions for 48 hours. These films (typically about $0.5 \mathrm{~mm}$ thick) were annealed (cured) at different temperatures $\left(50{ }^{\circ} \mathrm{C}, 60{ }^{\circ} \mathrm{C}, 70{ }^{\circ} \mathrm{C}\right.$, and $\left.80{ }^{\circ} \mathrm{C}\right)$ for 6 hours and then vacuum dried at $80{ }^{\circ} \mathrm{C}$ for an additional 6 hours. The optimal annealing temperature (confirmed by DSC) was found to be $60{ }^{\circ} \mathrm{C}$. The vacuum dried films were stored in a desiccator at room temperature.

Characterization. FTIR was performed using an Impact 400D (Nicolet, Madison, WI) to identify the polyurethane and nanocomposite structures. 32 scans at a $4-\mathrm{cm}^{-1}$ resolution were collected in the transmittance mode for each FT-IR spectra. A Hitachi model H-7500 transmission electron microscope (TEM) was used to examine the morphology of the films. The samples for TEM analysis were first prepared by placing the nanocomposite films in epoxy capsules and curing the epoxy at 70 ${ }^{\circ} \mathrm{C}$ for $24 \mathrm{~h}$ in an oven. The cured epoxies containing nanocomposite films were then microtomed with a diamond knife into 70-90-nm-thick slices at $-100{ }^{\circ} \mathrm{C}$. Finally, a 3-nm-thick carbon layer was deposited on these slices for TEM observation. The $T_{\mathrm{g}}$ was measured at $4 \mathrm{~Hz}$ with a dynamic mechanical thermal analyzer (DMTA MK III, Rheometrics Scientific, Inc., Surrey, UK) with a heating rate of $3{ }^{\circ} \mathrm{C} / \mathrm{min}$. The dimension of the film sample was $5 \times 5 \times 0.2\left(\mathrm{~mm}^{3}\right)$. For the water sorption tests, the films were immersed in water for $24 \mathrm{~h}$ at $25{ }^{\circ} \mathrm{C}$ and the water uptake was determined from the weight increase according to the following equation:

$$
\text { Water uptake }(\%)=\frac{\mathrm{W}-\mathrm{W}_{0}}{\mathrm{~W}_{0}} \quad \mathrm{X} 100
$$

where, $\mathrm{W}_{0}$ is the weight of the dried film and $\mathrm{W}$ is the weight of the film after being immersed in water. The mechanical properties were measured at room temperature with a Universal testing machine according to ASTM D 638 specifications. A crosshead speed of $50 \mathrm{~mm} / \mathrm{min}$ was used to determine the ultimate tensile strength and Young's modulus, and the elongation at break (\%) for all 
the samples. To evaluate the effect of swelled water on mechanical properties the film was dipped in water for $24 \mathrm{~h}$. The wet films were also dried fully (about $48 \mathrm{~h}$ at $60{ }^{\circ} \mathrm{C}$ ) to remove the swelled water, and measured the mechanical properties. The $T_{\mathrm{m}}$ and heat of fusion of the film was analyzed by differential scanning calorimetry (DSC, model 220C, Seiko, Chibas, Japan).

\section{Results and Discussion}

The modification of the CNTs and the subsequent fabrication of nanocomposites are shown in Scheme 1. The compositions of conventional WBPU, WBPU/CNT nanocomposites, WBPU/HMMM and WBPU/HMMM-CNT nanocomposites are summarized in Table 1. The functionalized CNT, HMMM-CNT, WBPU, WBPU/CNT, WBPU/HMMM and WBPU/HMMMCNT all were characterized by FTIR, (see Fig. 1 and 2).

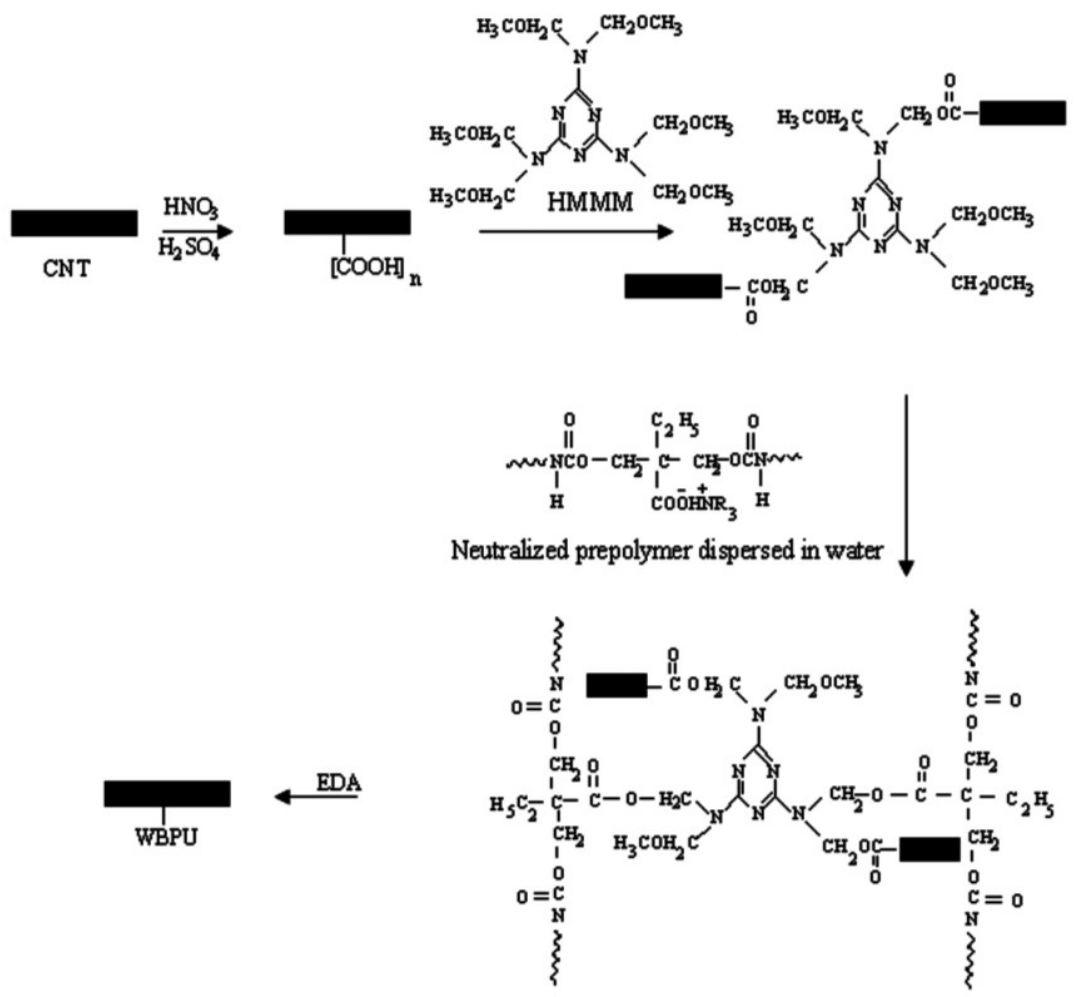

Scheme 1. Chemical route for the modification of CNT, crosslinking reaction and fabrication of WBPU/HMMM-CNT nanocomposite. 
In Fig. 1A, two prominent absorption bands appear at 1726 and $3434 \mathrm{~cm}^{-1}$ for the acid-treated CNTs. The peak at $1726 \mathrm{~cm}^{-1}$ is assigned to the characteristic acid carbonyl $(\mathrm{C}=\mathrm{O})$ stretching, and the broad peak around $3434 \mathrm{~cm}^{-1}$ is identified as the $\mathrm{O}-\mathrm{H}$ stretching mode in carboxylic acid groups [16]. The peak around $1580 \mathrm{~cm}^{-1}$ corresponds to the vibration mode of carbon skeleton of CNTs [16]. All of these peaks indicate that carboxylic acid groups are produced on the surface of CNTs after acid treatment. For HMMM-CNTs, two new peaks appeared at 2927 and $2846 \mathrm{~cm}^{-1}$ and assigned as the $\mathrm{C}-\mathrm{H}$ stretching mode of methylene in HMMM (not shown); compared with acidtreated CNTs there is an additional peak at $1647 \mathrm{~cm}^{-1}$, which we attributed to the new carbonyl $(\mathrm{C}=$ O) stretching attached to the HMMM (Fig. 1B). However, the characteristic acid carbonyl $(\mathrm{C}=\mathrm{O})$ peak at $1726 \mathrm{~cm}^{-1}$ does not fully disappear implying that some unreacted carboxylic acid groups still remain on the CNT surface. The peak at $1566 \mathrm{~cm}^{-1}$ is associated with $\mathrm{N}-\mathrm{H}$ stretching and the vibration of the carbon skeleton in CNTs, and the peak around $1232 \mathrm{~cm}^{-1}$ is attributable to the C-N stretching. There are also some additional bands at $1551,1482,815$ and $744 \mathrm{~cm}^{-1}$ corresponding to activity of the melamine derivative $[17,18]$. The band at $815 \mathrm{~cm}^{-1}$ is due to the out-of-plane ring deformation and another intense band around $1551 \mathrm{~cm}^{-1}$ is attributable to the $\mathrm{N}-\mathrm{C}-\mathrm{N}$ bending and ring deformation. The presence of a peak at $1482 \mathrm{~cm}^{-1}$ confirms the existence of some unreacted methoxy group in the HMMM-CNT dispersion [5]. Here, HMMM-CNT was prepared with a fixed amount of HMMM and a varied amount of CNT. The reactivity of the methoxy group of HMMM with CNT-COOH is unclear presently; additionally, their exact stoichiometric ratio is not known. Consequently, more studies are needed for better understanding of the mechanism. The spectrum of the WBPU (Fig. 2A) shows the characteristic polyurethane peaks, including the N-H peak at 3321 $\mathrm{cm}^{-1}$ and the $\mathrm{C}=\mathrm{O}$ peak at $1707 \mathrm{~cm}^{-1}[1,2]$. The spectrum of the WBPU/CNT nanocomposite is shown in Fig. 2B. There are no noticeable differences between the spectra of WBPU and WBPU/CNT. To identify the CNT dispersion in WBPU/CNT nanocomposite we used transmission electron microscopy (TEM), which is being described in next section. The IR spectrum of crosslinked WBPU/HMMM is also shown in Fig. 2C. The characteristic polyurethane peaks (the 
aforementioned $\mathrm{NH}$ and $\mathrm{CO}$ peak) as well as the melamine peak in the spectrum confirm that WBPU/HMMM is indeed crosslinked $[4,5]$. To identify the crosslinking reaction of HMMM-CNT with the carboxyl acid salt group of WBPU, the IR spectra were also analyzed (Fig. 2D). The IR spectrum of crosslinked WBPU/HMMM-CNT nanocomposite shows all the characteristic polyurethane peaks, including the $\mathrm{N}-\mathrm{H}$ peak at $3321 \mathrm{~cm}^{-1}$ and the $\mathrm{C}=\mathrm{O}$ peak at $1707 \mathrm{~cm}^{-1}$ for the urethane group of polyurethane as well as melamine characteristic peaks at 1551, 1482, 815 and 744 $\mathrm{cm}^{-1}[18]$.
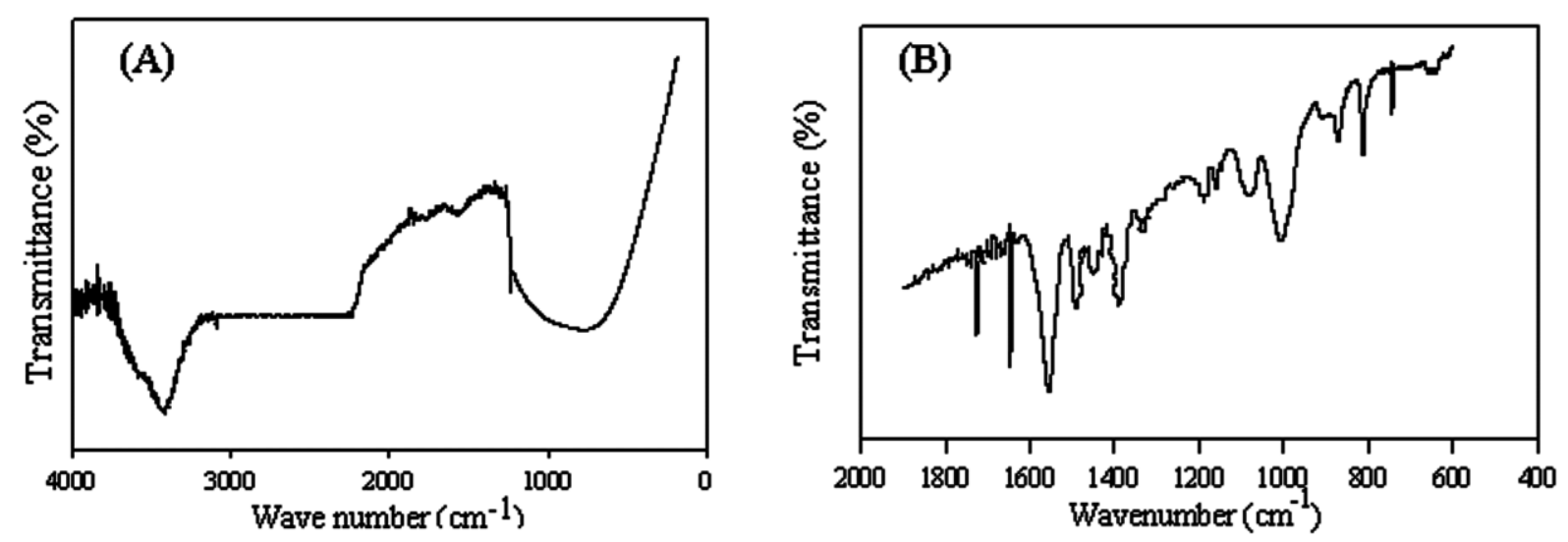

Fig. 1. FTIR spectra of (A) functionalized CNT and (B) HMMM-CNT (0.50 wt \%). 


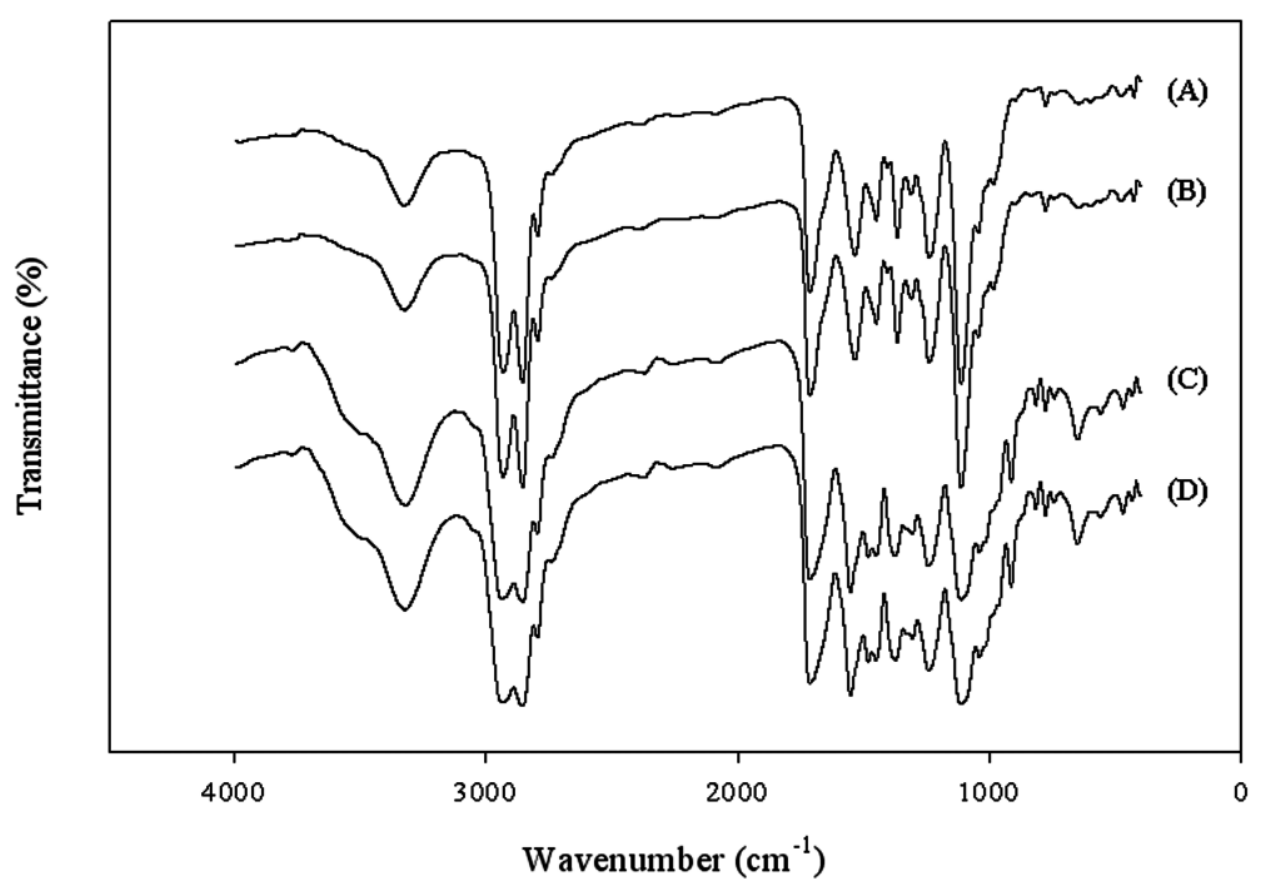

Fig. 2. FTIR spectra of (A) WBPU, (B) WBPU/CNT (0.5 wt\%), (C) WBPU/HMMM (5.0 wt \%) and (D) WBPU/HMMM-CNT (0.75 wt\%).

To identify the CNTs and their dispersion in WBPU/CNT nanocomposites and crosslinked WBPU/HMMM-CNT nanocomposites, we analyzed TEM images of the respective films. Generally, the efficiency of the CNTs in the matrix polymer is primarily determined by the degree of their dispersion. It has been reported that the properties of tensile strength, Young's modulus, adhesive strength, thermal stability and conductivity all increased with increasing CNT content up to the optimum value in WBPU/CNT nanocomposite $[10,12]$. The optimal homogeneous CNT content was 0.50 and $0.75 \mathrm{wt} \%$ for WBPU/CNT and WBPU/HMMM-CNT nanocomposite, respectively. Above the optimal content, the CNTs mainly aggregated in both nanocomposites (Fig. 3). CNTs have a tendency to aggregate in nanocomposites due to van der Waals interactions between the nanotubes [19]. This interaction can be broken by different methods such as importing various functional groups to the CNT surface, crosslinking CNT functional groups, and increasing the interaction within the carboxylic acid salt groups [10, 12, 20]. In HMMM-CNT, the CNT (carboxylic 
group) reacted with HMMM-methoxy group and thus made a distance between the CNT tubes. When the HMMM-CNT crosslinked with carboxylic acid salt group in WBPU/HMMM-CNT nanocomposite, the CNT keep the distance between the tubes and thus decrease the tendency of interaction between CNT tubes. This resulted in higher CNT loading $(0.75 \mathrm{wt} \%)$ in crosslinked WBPU/HMMM-CNT nanocomposites, which represents an increase in homogenous CNT loading of about $50 \%$ comparing to conventional WBPU/CNT nanocomposite.
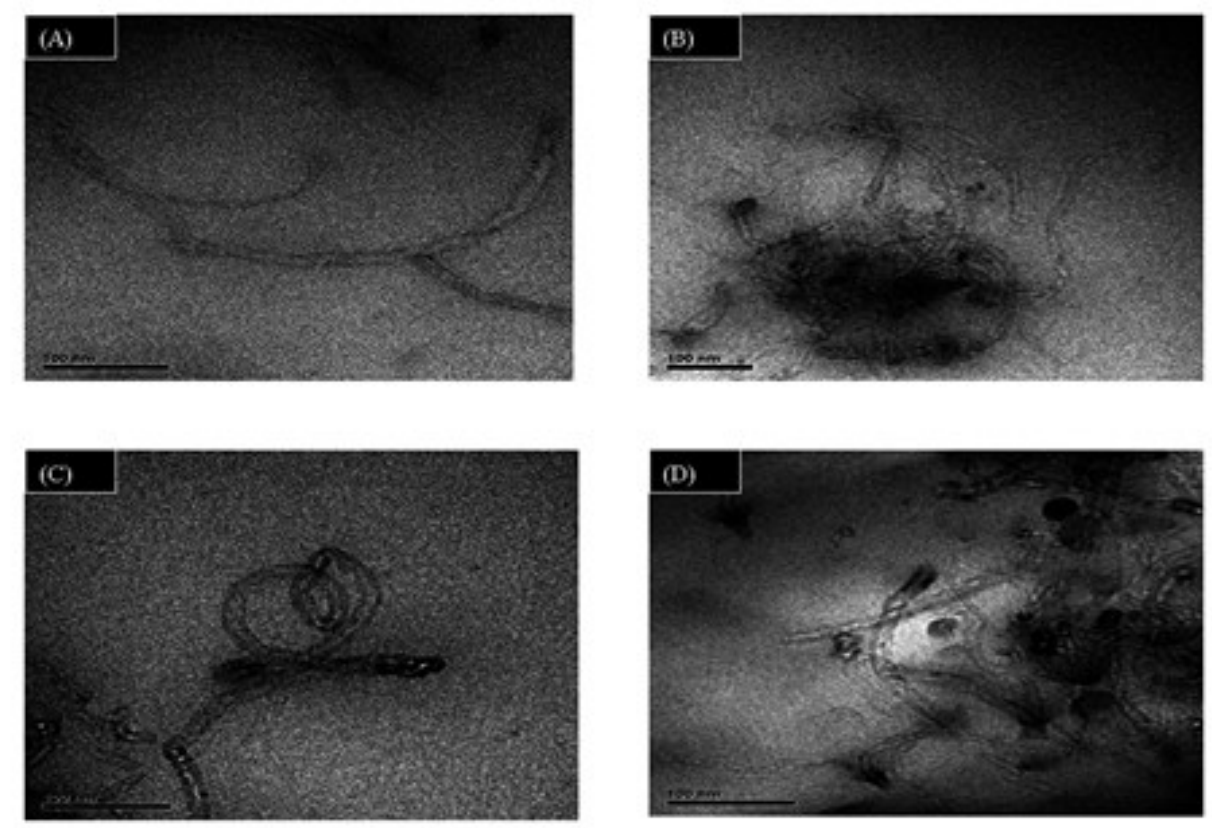

Fig. 3. TEM image of various nanocomposite films (A) WBPU/CNT $(0.5 \mathrm{wt} \%)$, (B) WBPU/CNT (0.75 wt\%), (C) WBPU/HMMM-CNT (0.75 wt\%) and (D) WBPU/HMMM-CNT (1.0 wt\%).

The properties of WBPU, crosslinked WBPU/HMMM and their nanocomposites depend on the glass transition temperature $\left(T_{\mathrm{g}}\right)$ values of the soft and hard segments $[3,10]$. We measured their $T_{\mathrm{g}}$ by DMTA analysis. The resulting $T_{\mathrm{g}}$ values are summarized in Table 2 . There were two peaks (Fig. 4) which can be ascribed to the $T_{\mathrm{g}}$ of the soft and hard segments. The dominant peak at around 108$110{ }^{\circ} \mathrm{C}$ represents the $T_{\mathrm{g}}$ of the hard segment and the weak broad peak at around $-52{ }^{\circ} \mathrm{C}$ to $-48{ }^{\circ} \mathrm{C}$ represents the $T_{\mathrm{g}}$ of the soft segment. The $T_{\mathrm{g}}$ values of both segments in WBPU/HMMM are higher than those in pristine WBPU (see Table 2). This can be ascribed to the crosslinking of the methoxy 
group of HMMM with the carboxylic acid salt group in WBPU [4]. The $T_{\mathrm{g}}$ values in the WBPU/CNT nanocomposite are also higher than those for pristine WBPU; and also $T_{\mathrm{g}}$ values increase with increasing CNT content. The high $T_{\mathrm{g}}$ value at small CNT loading may have resulted from the strong interaction between the CNT and the polymer matrix [10]. With inclusion of HMMM-CNT in nanocomposite, the $T_{\mathrm{g}}$ values increased significantly compared to those for pristine WBPU, WBPU/HMMM, and WBPU/CNT. The crosslinking as well as the CNT reinforcement further increased the $T_{\mathrm{g}}$ values of the nanocomposites. The increase in $T_{\mathrm{g}}$ values demonstrates the decreased amount of free volume $[20,21]$ in crosslinked WBPU/HMMM-CNT nanocomposites. The free volume is an important parameter for polymers as the water resistance of polymer films varies with the amount of free volume. It is reported that the water resistivity of polymer films increases as the amount of free volume decreases [22]. Therefore, the synthesized crosslinked WBPU/HMMMCNT nanocomposite can be used as a protective resin for water.

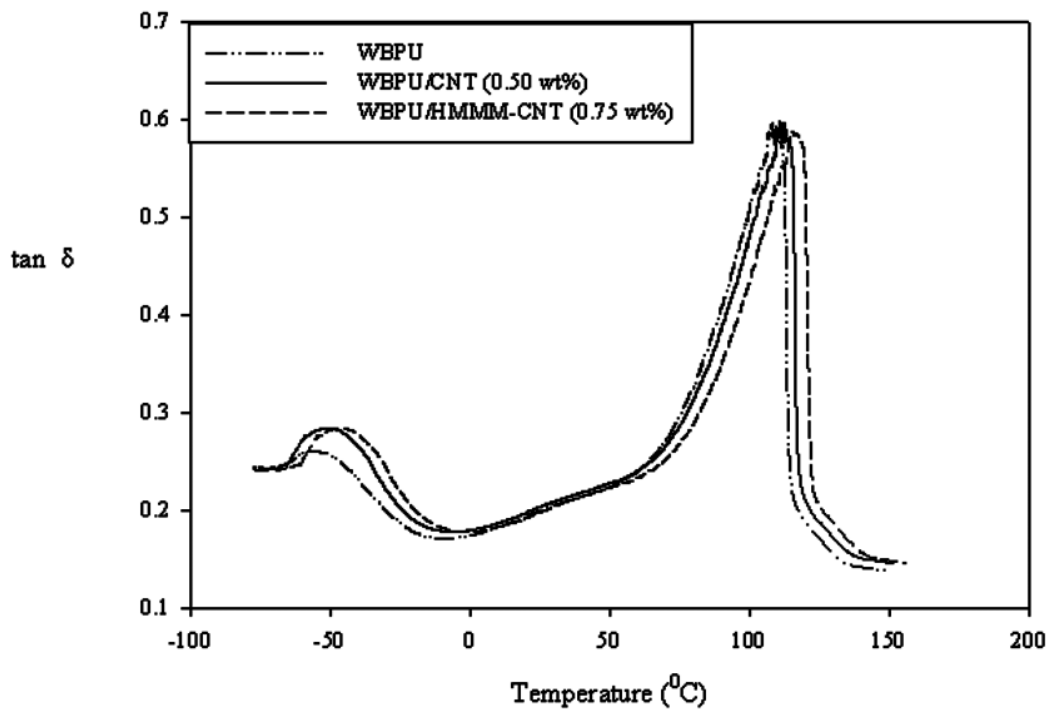

Fig. 4. $\tan \delta$ of WBPU, WBPU/CNT (0.5 wt $\%)$, and WBPU/HMMM-CNT $(0.75 \mathrm{wt} \%)$ crosslinked nanocomposite films . 
The viscoelastic properties of the films were also characterized by using DMTA analysis. Fig. 5 shows the storage module $\left(E_{0}\right)$ as a function of temperature of polymer with different compositions. These curves show three different zones: (i) at low temperature up to about $-60{ }^{\circ} \mathrm{C}$ is the zone of the glassy region, in which the storage modulus is high and it does not vary as temperature increases; (ii) the glass transition region and (iii) the rubber plateau followed by a drop of the storage modulus due to the melting of the soft segments. The WBPU, WBPU-HMMM, WBPU/CNT (with different CNT) and WBPU/HMMM-CNT nanocomposite (with different CNT/HMMM-CNT contents) show relatively similar curves (not shown). However, the storage modulus of WBPU/HMMM-CNT nanocomposites is higher than this for any of conventional WBPU, WBPU/HMMM, and WBPU/CNT nanocomposites. This might be due to the higher stiffening effect of jointly rigid CNT and crosslinking HMMM. The higher storage modulus of WBPU/HMMM-CNT nanocomposites implies that the molecular motions of these polymers chain are restricted than this for any other polymers (WBPU, WBPU/HMMM, WBPU/CNT) [10]. It is reported elsewhere that the water resistivity of polymer film decreased by increasing stiffness of polymer chain [23]. Thus the WBPU/HMMM-CNT nanocomposites can be used as a water protecting resin.

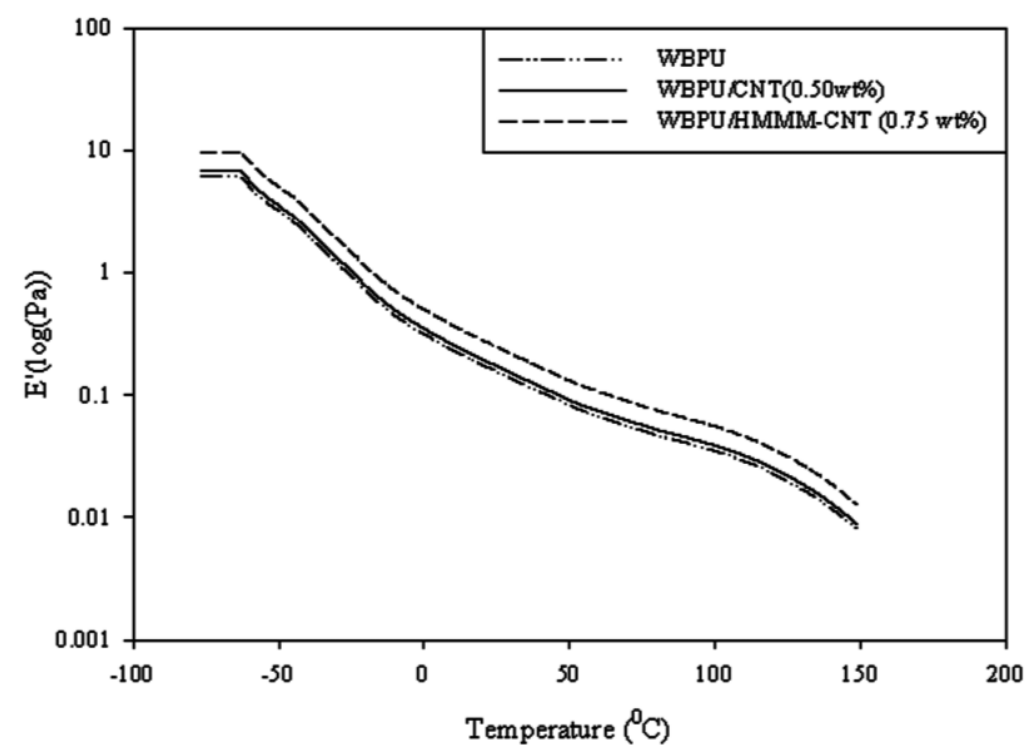

Fig. 5. Storage modulus of WBPU, WBPU/CNT (0.5 wt\%) nanocomposites and WBPU/HMMMCNT (0.75 wt \%) cross-linked nanocomposite films . 
Determining the amount (\%) of swelling in a polymer after being in water is a good method to determine its water resistance. All of the films had a tendency to swell after being dipped in water (see Table 2). However, the swelling rate was different for each sample. The crosslinked WBPU/HMMM-CNT nanocomposites, the crosslinked WBPU/HMMM and the conventional WBPU/CNT nanocomposites all showed higher water resistivity by absorbing less water comparing to this for conventional WBPU. The crosslinked WBPU/HMMM showed higher water resistivity due to the compact structure of the films made by crosslinking [23]. The conventional WBPU/CNT nanocomposites also showed higher water resistance due to having a structure with less free volume. The maximum water resistance of the nanocomposites was found at an optimal CNT content of 0.50 $w t \%$. At this concentration, the nanocomposites may have the least free volume and maximally compacted structure. Crosslinking (from HMMM) and reinforcement (from CNT) enable the crosslinked WBPU/HMMM-CNT nanocomposites to have the highest water resistance. Among all of the samples, due to having the highest number of stiff molecular chains for CNT reinforcement and HMMM crosslinking and the lowest amount of free volume (confirmed by DMTA), the water uptake (\%) was lowest for the film with the optimal HMMM-CNT content (Table 2), i.e., the water resistivity was highest in crosslinked WBPU/HMMM-CNT nanocomposites with the optimal HMMM-CNT content.

The mechanical properties are summarized in Table 3. The tensile strength and Young's modulus values are lower for conventional WBPU comparing to this for any of WBPU/CNT, WBPU/HMMM and WBPU/HMMM-CNT. The crosslinked WBPU/HMMM showed higher tensile strength as a result of crosslinking between the consecutive chains [22]. The tensile strength and Young's modulus values also increased with increasing CNT content and the tensile strength was highest at the optimal homogeneous CNT content $(0.50 \mathrm{wt} \%)$. The tensile strength and Young's modulus of crosslinked WBPU/HMMM-CNT nanocomposites were the maxima among all of the samples (up to the optimal HMMM-CNT content) comparing to those for pristine WBPU, crosslinked WBPU/HMMM and WBPU/CNT nanocomposite films. The elongation at break $(\%)$ decreased 
slightly with increasing HMMM-CNT content up to the optimal content; above the optimal point, the elongation at break (\%) decreased rapidly with increasing HMMM-CNT content. The higher tensile strength and Young's modulus of the crosslinked WBPU/HMMM-CNT nanocomposite film were observed with a optimum HMMM-CNT content due to the strong interaction between the CNTs and the polymer as well as due to polyurethane crosslinking between the two chains $[10,12]$. The lower tensile strength and Young's modulus values of the nanocomposite film with a HMMM-CNT concentration above the optimal content is due to the aggregation of excess CNT in crosslinked WBPU/HMMM-CNT nanocomposite film [10, 12]. At this composition [WBPU/HMMM-CNT $(0.75 \mathrm{wt} \%)]$, the tensile strength and Young's modulus were, respectively, $48 \%$ and $400 \%$ higher than those for pristine WBPU. We evaluated the mechanical properties of the films after they were dipped in water and after they were dried. The tensile strength, Young's modulus and elongation at break (\%) decreased after being in water for all of the films. However, the rate at which these mechanical properties decreased were not the same. The values for conventional WBPU film decreased most rapidly due to swelling from the water compared to the values for crosslinked WBPU/HMMM and WBPU/CNT nanocomposite and to those for crosslinked WBPU/CNT-HMMM nanocomposites. The film with strength parameters that decreased $(8 \%)$ the slowest was the crosslinked WBPU/CNT-HMMM nanocomposite with optimal HMMM-CNT content, as expected from its minimal swelling from the water. Thus, the WBPU/HMMM-CNT $(0.75$ wt $\%)$ nanocomposite had the highest mechanical resistance (tensile strength and Young's modulus) under water among all of the samples.

We also checked the mechanical properties of the samples after they were dried (see Table 3). Interestingly, the tensile strength, Young's modulus and elongation at break (\%) values for each crosslinked WBPU/HMMM-CNT nanocomposite films recovered maxima after drying and were comparable to those for untreated samples. The conventional WBPU film had the lowest values to recover the tensile strength and Young's modulus. The dried crosslinked WBPU/HMMM and WBPU/CNT nanocomposites had higher mechanical strength values than the wet samples but 
significantly lower values than the untreated samples. The fact that crosslinked WBPU/HMMMCNT nanocomposites recovered highest in strength implies that these were less affected by water. The tensile strength was almost fully recovered with optimal HMMM-CNT content in crosslinked WBPU/HMMM-CNT nanocomposite implies that this film is barely affected by water among all of the films and maintained this original mechanical strength.

We applied the DSC technique (Fig. 6) to analyze the structure of the films under the three conditions (untreated, wet, and dried). We selected one sample (the sample which has the optimal content) from each series to analyze its $T_{\mathrm{m}}$ and heat of fusion. The resulting values are summarized in Table 4. We observed that the untreated, wet and dried crosslinked WBPU/HMMM-CNT nanocomposite film had similar DSC curves and $T_{\mathrm{m}}$ values, except for one small peak appearing at 0 ${ }^{\circ} \mathrm{C}$ for water in the wet sample. For the conventional WBPU, the $T_{\mathrm{m}}$ fully disappeared at wet and dried conditions. Again, the wet sample had a large peak at $0{ }^{\circ} \mathrm{C}$ for water, and disappeared for the dried sample. The dried sample also did not yield a melting temperature, implying that the crystalline structure of conventional WBPU was damaged in presence of water and was not recovered after drying $[24,25]$. 

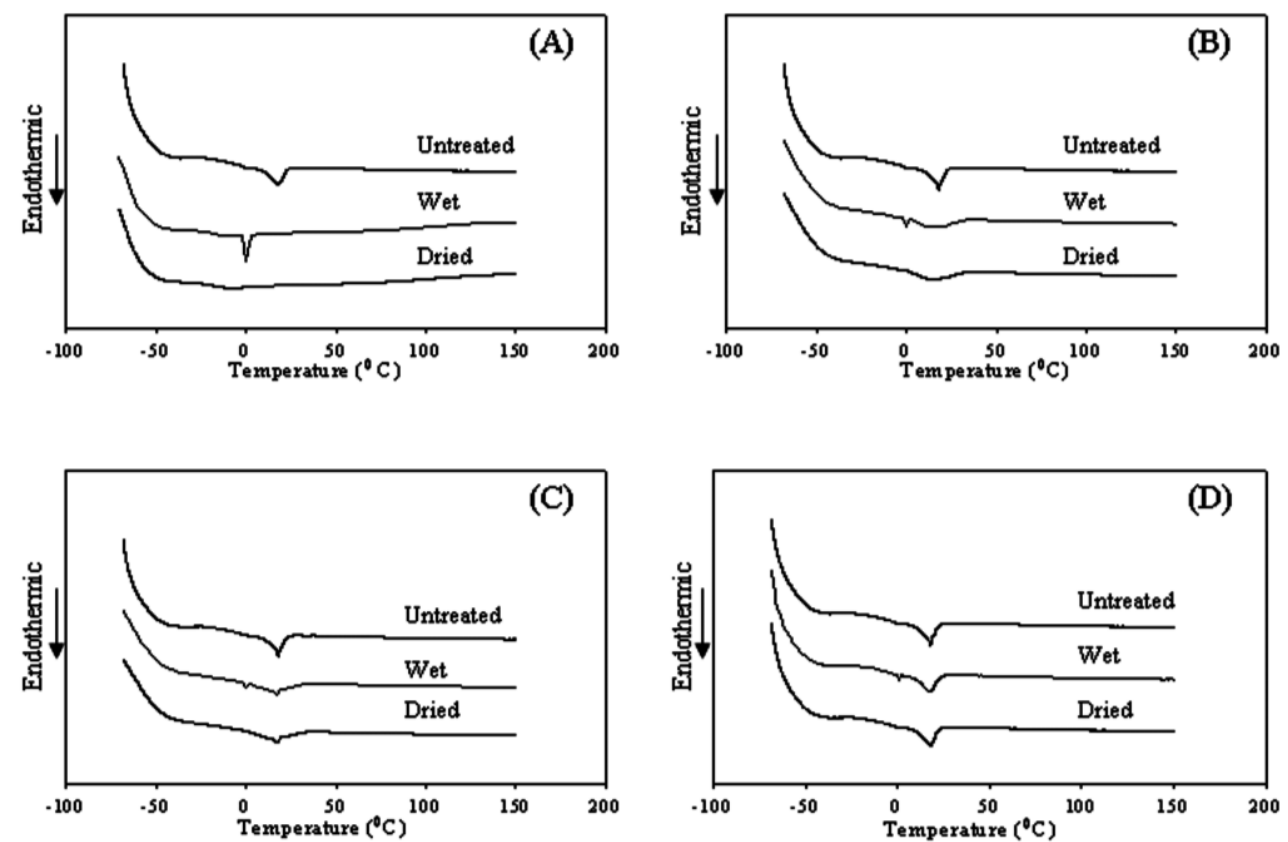

Fig. 6. DSC of conventional (A) WBPU, (B) cross-linked WBPU/HMMM, (C) WBPU/CNT nanocomposites and (D) WBPU/HMMM-CNT (0.75 wt\%) nanocomposite films at three stages (untreated, wet and dried).

The WBPU/HMMM and WBPU/CNT samples had low heat of fusion (see Table 4) and confirmed their crystalline structures also being destroyed by water. The synthesized crosslinked WBPU/HMMM-CNT maintained its original structure even under the adverse conditions of being dipped in water and then dried. It is well known that the absorbed water in polymer can be remained in three states such as free water, freezable bound water and non-freezable bound water [26, 27]. The endothermic peak at $0{ }^{\circ} \mathrm{C}$ is only for free water. After drying the peak at $0{ }^{\circ} \mathrm{C}$ was fully disappeared for conventional WBPU. Disappearance of melting temperature also implied that some was boundwater which damages the crystalline structure. Thus the polymer structure was changed to almost amorphous. The $T_{\mathrm{m}}$ of crosslinked WBPU/HMMM-CNT nanocomposite still remains in wet and dry condition. Moreover, the endothermic peak for water was disappeared by heating implied that the absorbed water in this nanocomposite mainly free water which could not damage the crystalline 
structure. Thus the tensile strength and Young's modulus were almost similar at three stages. The tensile strength and Young's modulus at dried condition of crosslinked WBPU/HMMM and conventional WBPU/CNT were intermediate between untreated and wet condition. For both samples some water might be bound water which was reflected by decreasing the heat of fusion. Hence the tensile strength and Young's modulus values were also in between at untreated and wet condition for those values of dried samples.

The above discussion suggests that the synthesized crosslinked WBPU/HMMM-CNT nanocomposite can absorb less water (mainly free water) and hence the tensile strength was slightly affected after dipped in water. The absorbed free water cannot damage the crystalline structure of this crosslinked nanocomposite. Generally the equilibrium water uptake by any polymeric system involves sorption, diffusion, and permeation processes. Sorption is a surface phenomenon and indicates the affinity of the material to water molecules, which is dependent on the chemical characteristics of the sample surface. Diffusion is the process where the penetrant moves through the polymer depending on the polymer relaxation process. The permeability represents the combined effect of the diffusivity as well as the solubility or sorptivity of the penetrant in a polymer. Besides, the uptake of any solvent by a polymeric system depends on the temperature, molecular weight and size of the solvent molecule, the cross-linking density and composition of the nanocomposites, the polymer/solvent interaction, as well as the microstructure of the nanocomposites [28-30]. The less water swelling of synthesized crosslinked nanocomposite might be due to less diffusion coefficient and permeability coefficient. The decrease in diffusion coefficient with CNTs can be ascribed to the resistance to water diffusion due to CNTs. Permeability coefficient decreases with CNTs as they hinder the movement of water molecules between the polymer chains. The cross-linked system made the polymer surface less interactive with the water molecules. Moreover, the swelled water is mainly free water, which could not change the molecular structure as well as crystallinity and this water can be removed easily by drying. Therefore, the cross-linked nanocomposites have significantly lower sorption owing to the combined effect of cross-linking and presence of CNTs. Combination of these 
factors made the crosslinked WBPU/HMMMCNT nanocomposite less water sensitive, and the mechanical properties were very slightly affected after dipped in water and recovered the values after drying. We compare schematically the water swelling of conventional WBPU and crosslinked WBPU/HMMM-CNT nanocomposite in Fig. 7. The synthesized crosslinked WBPU/HMMM-CNT nanocomposite maintained its original mechanically strong structure even under the adverse conditions of being dipped in water and then dried and hence has the highest water resistance as well as perform tensile strength similar to untreated sample. It can thus be considered to be a potential water-resistant coating for various applications.
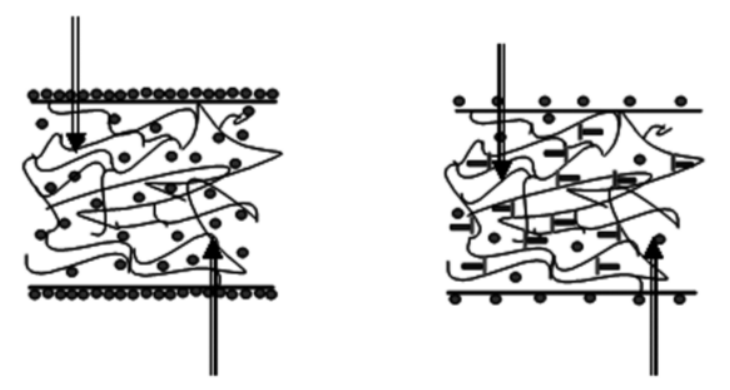

Conventional WBPU

Crosslinkable WBPU/HMMM-CNT nanocomposite

(High sorption, diffusion and permeation)

(Reduced sorption, diffusion and permeation)

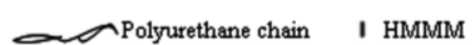

- CNT - Water molecules

Fig. 7. Comparison of water swelling of conventional WBPU and WBPU/HMMM-CNT nanocomposites.

\section{Conclusion}

We have successfully synthesized crosslinked WBPU/HMMM-CNT nanocomposites and compared their properties with those for conventional WBPU, crosslinked WBPU/HMMM, and WBPU/CNT nanocomposite. The tensile strength and Young's modulus of the crosslinked WBPU/HMMM-CNT nanocomposite film increased $48 \%$ and $400 \%$ respectively, compared to those fo conventional 
WBPU. Only the crosslinked WBPU/HMMM-CNT nanocomposite showed the significant mechanical properties (tensile strength and Young's modulus) in both wet and dry condition. Due to its higher water resistance as well as higher tensile strength and Young's modulus even after being in water, the crosslinked WBPU/HMMM-CNT nanocomposite can be treated a potential waterresistant coating material and can be applied to many applications to broadened environmentally friendly coating.

\section{Acknowledgement}

Authors are thankful to Center of Research Excellence in Corrosion Science and Center of Research Excellence in Nano Technology of King Fahd University of Petroleum and Minerals, for providing excellent research facilities.

\section{References}

[1] M.M. Rahman, H.D. Kim, Synthesis and characterization of waterborne polyurethane adhesives containing different amount of ionic groups (I), J. Appl. Polym. Sci. 102 (2006) $5684-5691$.

[2] M.M. Rahman, A. Hasneen, W.K. Lee, K.T. Lim, Preparation and properties of Sol-gel Waterborne Polyurethane Adhesives, J. Sol-gel. Sci. Technol. 67 (2013) 473-479.

[3] J.K. Yun, H.J. Yoo, H.D. Kim, Preparation and properties of waterborne polyurethaneurea/sodium alginate blends for high water vapor permeable coating materials, J. Appl. Polym. Sci. 105 (2007) 1168-1176. 
[4] K. Mequanint, R. Sanderson, Nano-structure phosphorus-containing polyurethane dispersions: synthesis and crosslinking with melamine formaldehyde resin, Polymer 44 (2003) 2631-2639.

[5] M.M. Rahman, E.Y. Kim, J.Y. Kwon, H.J. Yoo, H.D. Kim, Cross-linking reaction of waterborne polyurethane adhesives containing different amount of ionic groups with hexamethoxymethyl melamine, Int. J. Adhes. Adhes. 28 (2008) 47-54.

[6] Y.S. Kwak, E.Y. Kim, B.H. Yoo, H.D. Kim, Preparation and properties of waterborne poly(urethane urea)s for adhesives: The effects of the 2,2-bis(hydroxylmethyl)propionic acid content on the properties, J. Appl. Polym. Sci. 94 (2004) 1743-1751.

[7] J.E. Yang, Y.H. Lee, Y.S. Koo, Y.J. Jung, H.D. Kim, Preparation and properties of waterborne poly(urethane-urea) ionomers effect of the type of neutralizing agent, Fiber. Polym. 3 (2002) 97-102.

[8] B.S. Kim, B.K. Kim, Enhancement of hydrolytic stability and adhesion of waterborne polyurethanes, J. Appl. Polym. Sci. 97 (2005) 1961-1969.

[9] M.M. Rahman, H.D. Kim, Effect of polyisocyanate hardener on adhesive force of waterborne polyurethane adhesives, J. Appl. Polym. Sci. 104 (2007) 3663-3669.

[10] J.Y. Kwon, H.D. Kim, Comparison of the properties of waterborne polyurethane/multiwalled carbon nanotube and acid-treated multiwalled carbon nanotube composites prepared by in situ polymerization, J. Polym. Sci. Part A: Polym. Chem. 43 (2005) 3973-3985.

[11] J.H. Huh, M.M. Rahman, H.D. Kim, Properties of Waterborne Polyurethane/Clay Nanocomposite Adhesives, J. Adhes. Sci. Technol. 23 (2009) 739-751. 
[12] M.M. Rahman, E.Y. Kim, K.T. Lim, W.K. Lee, Morphology and Properties of Waterborne Polyurethane/CNT Nanocomposite Adhesives with Various Carboxyl Acid Salt Groups, J. Adhes. Sci. Technol. 23 (2009) 839-850.

[13] X. Hong, Q. Chen, M. Chen, J. Chen, H. Liu, A novel coating by hybrid polymerization, Prog. Org. Coat. 45 (2002) 165-171.

[14] X. Hong, The effect of HMMM in a hybrid polymerization comprising free radical polymerization, Prog. Org. Coat. 26 (1995) 121-129.

[15] P. Marino, C. Lowe, M.-L. Abel, J.F. Watts, Surface characterization of polyester resins formulated with different cross-linking agents, Surf. Interface Anal. 40(3) (2008) 137-141.

[16] L. Liu, Y. Qin, Z.-X. Guo, D. Zhu, Reduction of solubilized multi-walled carbon nanotubes, Carbon 41 (2003) 331-335.

[17] Y.-L. Wang, A.M. Mebel, C.-J. Wu, Y.-T. Chen, C.-E. Lin, J.-C. Jiang, IR spectroscopy and theoretical vibrational calculation of the melamine molecule, J. Chem. Soc Faraday Trans. 93 (1997) 3445-3451.

[18] P.J. Larkin, M.P. Makowski, N.B. Colthup, L.A. Flood, Vibrational analysis of some important group frequencies of melamine derivatives containing methoxymethyl, and carbamate substituents: mechanical coupling of substituent vibrations with triazine ring modes, Vib. Spectrosc. 17 (1998) 53-71.

[19] Yu, X.; Kwon, E. A carbon nanotube/cement composite with piezoresistive properties, Smart Mater. Struct. 18 (2009) 055010:1-055010:5. 
[20] J.N. Deng, X.Q. Zhang, K. Wang, H. Zou, Q. Zhang, Q. Fu, Synthesis and properties of poly(ether urethane) membranes filled with isophorone diisocyanate-grafted carbon nanotubes, J. Membr. Sci. 288 (2007) 261-267.

[21] H. Xia, M. Song, Preparation and characterization of polyurethane-carbon nanotube composites, Soft Matter 1 (2005) 386-394.

[22] M.M. Rahman1, I. Lee, H.-H. Chun, H.D. Kim, H. Park, Properties of waterborne polyurethane-fluorinated marine coatings: The effect of different types of diisocyanates and tetrafluorobutanediol chain extender content, J. Appl. Polym. Sci. 131(4) (2014) 39905 (7 page).

[23] M.M. Rahman, H.D. Kim, W.K. Lee, Properties of crosslinked waterborne polyurethane adhesives with modified melamine: Effect of curing time, temperature, and HMMM content, Fiber. Polym. 10 (1) (2009) 6-13.

[24] R. Gref, Q.T. Nguyen, J. Rault, J. Néel, Etats des solvants absorbes dans des films semicristallins en alcool polyvinylique-I. Gonflement dans l'eau, Eur. Polym. J. 28(8) (1992) 1007-1014.

[25] R. Iwamoto, M. Miya, S. Mima, Determination of crystallinity of swollen poly(vinyl alcohol) by laser Raman spectroscopy, J. Polym. Sci. B. Polym. Phys. 17(9) (1979) 15071515.

[26] A. Higuchi, T. Iijima, DSC investigation of the states of water in poly(vinyl alcohol) membranes, Polymer 26 (1985) 1207-1211.

[27] A. Higuchi, T. Iijima, D.s.c. investigation of the states of water in poly(vinyl alcohol-coitaconic acid) membranes, Polymer 26 (1985) 1833-1837. 
[28] E. Southern, A.G. Thomas, Diffusion of liquids in crosslinked rubbers. Part 1, Trans. Faraday Soc. 63 (1967) 1913-1921.

[29] B. Alexandre, S. Marais, D. Langevin, P. Médéric, T. Aubry, Nanocomposite-based polyamide 12/montmorillonite: relationships between structures and transport properties, Desalination 199 (2006) 164-166.

[30] R. Stephen, S. Varghese, K. Joseph, Z. Oommen, S. Thomas, Diffusion and transport through nanocomposites of natural rubber (NR), carboxylated styrene butadiene rubber (XSBR) and their blends, J. Membr. Sci. 282 (2006) 162-170. 